INNOVATIONS IN PRIMARY CARE

\title{
Partnering Research Fellows and Clinicians in Practice Settings
}

\author{
Maria Ukhanova, $M D, P b D^{*}$ \\ Sonja Likumabuwa-Ackman, \\ Jobn Heintzman, MD, MPH \\ Ann Tseng, MD \\ MID, MPH \\ Jennifer DeVoe, MD, DPbil \\ Daisuke Yamashita, MD \\ Oregon Health \& Science University, Department of Family Medi- \\ cine, Portland, Oregon
}

Ann Fam Med 2019;17:86. https://doi.org/10.1370/afm.2333.

\section{THE INNOVATION}

At the front line of patient care, family physicians have questions about how to improve their practice or track an impact of ongoing initiatives, but often lack experience or time to seek answers in the clinical setting. Collaborations with researchers might be an effective way to answer relevant questions, but these partnerships are sporadic, and can be dominated by "top-down" research projects. 1-3 Using a researcher-in-residence model, we created the Oregon Health \& Science University (OHSU) Postdoctoral Research Fellowship in Family Medicine. We provide on-site research expertise for clinicians wanting to transform clinical questions into research and quality improvement projects, and to give researchers an immersion experience with clinical experts.

\section{WHO \& WHERE}

The OHSU Department of Family Medicine operates multiple primary care clinics in Oregon. To augment the traditional research fellowship experience, we embedded 2 postdoctoral fellows into 2 clinics in Portland. Fellows were newly graduated Public Health PhDs training for careers in academic primary care research.

\section{HOW}

Once a week, fellows spent a working day at the clinic, developing relationships with clinic leadership by attending meetings, shadowing staff, and keeping activity logs of clinicians' research interests. Fellows also held regular office hours (inperson and virtual) where clinicians could easily discuss ideas, regardless of developmental stage.

In the first year, part-time clinicians/faculty members with limited research experience leveraged fellows' knowledge, while fellows gained first-hand knowledge of clinical care and practice operation. Fellows helped formulate research questions and data

Conflicts of interest: authors report none.

*Corresponding author: ukhanova@ohsu.edu collection/analysis; clinicians identified study questions relevant to practice needs, facilitated medical interpretation of the findings, and were involved in research processes depending on time and interest. For instance, fellows assisted with literature review and project framing to evaluate a model for Hepatitis C treatment in primary clinics and assisted with an abstract submission to a national conference. At another clinic, the director collaborated with fellows to evaluate efforts to reduce employee burnout: the assessment of clinical quality metrics sparked the development of an interview guide to evaluate employee engagement at the clinic. One project paused after initial discussions/assistance due to lack of funding for data collection; however, fellows intend to conduct future data analysis.

\section{LEARNING}

Clinicians were open to the embedded researcher model. Fellows' presence motivated the clinicians to explore ideas; the fellows acted as a sounding board and lowered the barrier to formulate logical steps to approach tasks. In several cases, however, the transition from generating ideas to implementation stalled due to competing clinical responsibilities, lack of funding, the need for more senior researcher input, or uncertainty about the fellows' exact role in clinic projects (do they just advise, or help staff the project as well?). At a faculty retreat, some faculty expressed appreciation for the presence of the fellows, but were still unsure about how to best utilize them. Developing these relationships and roles across multiple clinics will take time. Next year, we will engage fellowship faculty more actively to foster project and relationship development. We will identify clinicians/co-medical staff who worked successfully with researchers who can serve as clinic "champions" for new fellows: eg, currently 2 clinics are launching program evaluations for opioid addiction treatment where fellows participate by interviewing clinical teams.

Embedded research fellows can be a bridge between research and clinical practice, connecting clinical and research faculty who often work in silos. Beyond academic departments, such a model could facilitate a culture of critical inquiry in clinical settings, provide clinicians access and tools to fulfill their scholarly curiosity and practice needs, establish structured partnerships and build research capacity in primary care. For community practices without direct or on-location academic affiliations, a virtual model might be beneficial, although this requires further development and evaluation.

Corresponding author contact information, submitted dates, key words, and references are available at http://www.AnnFamMed. org/content/17/1/86/suppl/DC1. 